\title{
LU60645GT and MA132843GT catalogues of Lunar and Martian impact craters developed using a Crater Shape-based interpolation crater detection algorithm for topography data
}

\author{
Goran Salamunićcar $^{\mathrm{a}, \mathrm{b}, *}$, Sven Lončarić ${ }^{\mathrm{b}}$, Erwan Mazarico ${ }^{\mathrm{c}, \mathrm{d}}$ \\ ${ }^{a}$ AVL-AST d.o.o., Av. Dubrovnik 10/II, 10020 Zagreb-Novi Zagreb, Croatia \\ ${ }^{\mathrm{b}}$ Faculty of Electrical Engineering and Computing, University of Zagreb, Unska 3, 10000 Zagreb, Croatia \\ ${ }^{\mathrm{c}}$ NASA Goddard Space Flight Center, Planetary Geodynamics Laboratory, Greenbelt, MD 20771, USA \\ ${ }^{\mathrm{d}}$ Massachusetts Institute of Technology, Department of Earth, Atmospheric and Planetary Sciences, Cambridge, MA 02139, USA
}

\section{A R T I C L E I N F O}

\section{Article history:}

Received 6 July 2011

Received in revised form

31 August 2011

Accepted 2 September 2011

Available online 16 September 2011

Keywords:

Moon

Mars

Surface

Cratering

Image processing

\begin{abstract}
A B S T R A C T
For Mars, 57,633 craters from the manually assembled catalogues and 72,668 additional craters identified using several crater detection algorithms (CDAs) have been merged into the MA130301GT catalogue. By contrast, for the Moon the most complete previous catalogue contains only 14,923 craters. Two recent missions provided higher-quality digital elevation maps (DEMs): SELENE (in 1/16 resolution) and Lunar Reconnaissance Orbiter (we used up to $1 / 512^{\circ}$ ). This was the main motivation for work on the new Crater Shape-based interpolation module, which improves previous CDA as follows: (1) it decreases the number of false-detections for the required number of true detections; (2) it improves detection capabilities for very small craters; and (3) it provides more accurate automated measurements of craters' properties. The results are: (1) LU60645GT, which is currently the most complete (up to $\sim D \geq 8 \mathrm{~km}$ ) catalogue of Lunar craters; and (2) MA132843GT catalogue of Martian craters complete up to $\sim D \geq 2 \mathrm{~km}$, which is the extension of the previous MA130301GT catalogue. As previously achieved for Mars, LU60645GT provides all properties that were provided by the previous Lunar catalogues, plus: (1) correlation between morphological descriptors from used catalogues; (2) correlation between manually assigned attributes and automated measurements; (3) average errors and their standard deviations for manually and automatically assigned attributes such as position coordinates, diameter, depth/diameter ratio, etc; and (4) a review of positional accuracy of used datasets. Additionally, surface dating could potentially be improved with the exhaustiveness of this new catalogue. The accompanying results are: (1) the possibility of comparing a large number of Lunar and Martian craters, of e.g. depth/diameter ratio and 2D profiles; (2) utilisation of a method for re-projection of datasets and catalogues, which is very useful for craters that are very close to poles; and (3) the extension of the previous framework for evaluation of CDAs with datasets and ground-truth catalogue for the Moon.

(c) 2011 Elsevier Ltd. All rights reserved.
\end{abstract}

\section{Introduction}

Recently, all the craters from the major currently available manually assembled catalogues have been merged into the MA57633GT catalogue with 57,633 known Martian impact-craters (Salamunićcar and Lončarić, 2008b). In addition, using our crater detection algorithm (CDA) and $1 / 128^{\circ}$ MOLA data, 57,592 previously uncatalogued craters have been identified resulting in the MA115225GT catalogue (Salamunićcar and Lončarić, 2010a). This catalogue has been additionally extended using: (1) MA75919T catalogue, which is the result of the CDA from Stepinski and Urbach (2008); (2) the CDA from our previous work (Salamunićcar

\footnotetext{
* Corresponding author at: AVL-AST d.o.o., Av. Dubrovnik 10/II, 10020 Zagreb-Novi Zagreb, Croatia. Tel.: +385 98890725.

E-mail addresses: gsc@ieee.org (G. Salamunićcar),

sven.loncaric@fer.hr (S. Lončarić), erwan.m.mazarico@nasa.gov (E. Mazarico).
}

and Lončarić, 2010a), wherein instead of the Canny we used ShenCastan edge detector; and (3) the CDA for optical images (Bandeira et al., 2007) for four selected regions of interest. This resulted in MA130301GT catalogue (Salamunićcar et al., 2011b), which is at the time of writing the most complete previously available public catalogue of Martian craters. By contrast, for the Moon the most complete previous catalogue contains only 14,923 craters (Rodionova et al., 1987). The size difference between Mars and the Moon only partially explains the difference, because on the Moon the average density of the largest craters is greater than on Mars according to size-frequency distribution of the largest craters, for which these catalogues are mostly complete (see below; Section 3.2 - the distribution of craters from corresponding catalogues). Two recent missions provided higher-quality digital elevation maps (DEMs): (1) SELENE laser altimetry (LALT) dataset in $1 / 16^{\circ}$ resolution (Araki et al., 2009); and (2) Lunar Reconnaissance Orbiter (LRO) Lunar orbiter laser altimeter (LOLA) dataset (Smith et al., 2010), wherein 
we used resolutions up to $1 / 512^{\circ}$. This was a motivation for our work on a considerably more complete catalogue of Lunar craters.

Research on CDAs is challenging for numerous reasons. On Mars, there are many different crater shapes depending on their interior morphologies (central peaks, peak rings, central pits, and wall terraces) and ejecta structures (pedestal, pancake, rampart, lobate, fluidized, radial or lunar-like, transitional or diverse) (Barlow et al., 2003). There is also a simple-complex transition from the smaller, mostly very circular bowl-shaped craters, to larger complex craters with central peaks, and to the largest multi-ring impact basins (Melosh and Ivanov, 1999). In general, CDAs are based on a large number of methods, including circle/ ellipse detection (Cooper, 2003; Cooper and Cowan, 2004; FloresMéndez and Suarez-Cervantes, 2009; Krøgli and Dypvik, 2010), probability volume created by template matching (Bandeira et al., 2007), machine-learning (Stepinski and Urbach, 2008), etc. An overview of 112 publications related to CDAs has been published in two recent papers (Salamunićcar and Lončarić, 2008a; Salamunićcar et al., 2011b). The list is constantly increasing, as is e.g. the work regarding feature selection and boosting (Ding et al., 2010) and entropic quadtrees (Vetro and Simovici, 2010). However, the state of the art in image-analysis/objectrecognition still does not offer an answer on how to create a CDA that is as robust as the scientific community would desire. Hence, it is not surprising that several research groups are working in this still young field, trying to achieve this goal. Several CDAs can process global datasets (Michael, 2003; Salamunićcar and Lončarić, 2010a; Stepinski and Urbach, 2008). The experience with our CDA is as follows: (1) it is necessary to manually process a large number of crater-candidates in order to considerably extend ground-truth (GT) catalogue; (2) improvements of detection capabilities for very small craters increase the number of detected craters from any given dataset; and (3) the inaccuracy in measurement of craters' properties including 2D profiles increases with the decrease in their size. This was a motivation for the work on the new Crater Shape-based interpolation, which considerably improves the previous CDA in addressing these issues.

The above led to the concurrent work on new catalogues and improvements of the previous CDA (Salamunićcar and Lončarić, 2010a). Resulting catalogues are: (1) MA132843GT for Mars; and (2) LU60645GT for the Moon, created using LALT (Salamunićcar and Lončarić, 2010b) and LOLA (Salamunićcar et al., 2011a) datasets.

The rest of this paper is organised as follows. In Section 2, the methods and datasets of this paper are presented in detail, as well as used and assembled datasets. The results are presented in Section 3, and the conclusion is given in Section 4. Readers mostly interested into the improved CDA from this paper should be primarily focused to Sections 2.1, 2.2, and 3.1, readers mostly interested into the resulting catalogues should be primarily focused to Sections $2.3,2.4$, and 3.2., while Section 3.3 demonstrates how new crater catalogues can be used in combination with the improved CDA which provides automated measurements, in order to outline differences between Martian and Lunar craters.

\section{Methods and datasets}

The developed methods and the datasets used are described in the following order:

- improvement of crater detection algorithm using new Crater Shape-based interpolation;

- utilisation of method for re-projection of datasets and catalogues;

- datasets used for crater detection and evaluation; and

- previous catalogues of Lunar craters.
The following are made available online (http://informatika. tvz.hr/index.php?pred=17461): the source code (Craters5_77.zip) of the interpolation-based CDA presented in this paper; the Topolyzer application, which implements methods for the evaluation of CDAs and the registration to ground-truth (GT) catalogues; and the catalogues LU60645GT and MA132843GT (as well as previous versions). In addition, the various datasets are available on request, in order to provide reproducibility of the methods presented in this paper and inter-comparison with other CDAs.

\subsection{Improvement of crater detection algorithm using new Crater Shape-based interpolation}

The CDA from this paper, which uses multi-resolution image analysis and the Crater Shape-based interpolation algorithm described in following paragraphs, is shown in Fig. 1. The algorithm is an extension of our previous work (Salamunićcar and Lončarić,

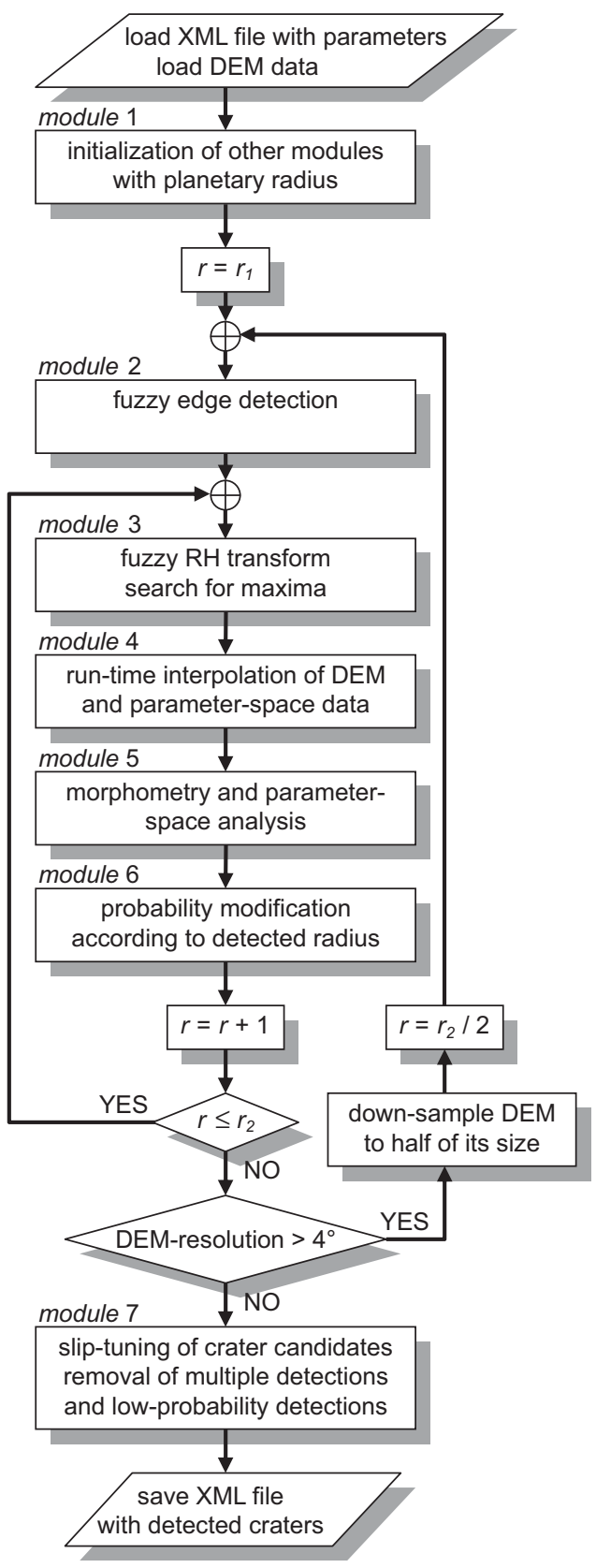

Fig. 1. Schematic flow diagram of overall organisation and processing steps for our CDA. 
2010a). The algorithm utilises fuzzy edge detection to extract crater features required for the fuzzy Radon-Hough ( $\mathrm{RH})$ transform search for maxima in the parameter space. Following this step, DEM and parameter-space data are interpolated at run-time in order to be used for morphometry measurements (of depth/diameter ratio, circularity, topographic-cross-profile, rim, central peak, and radial range where the crater is preserved) and parameter space analysis (the circularity of votes is higher at craters' centres than in the centres of false detections). The interpolation at run-time is used to dynamically calculate interpolated values on demand, therefore reducing memory requirements. Next, the previously computed probability that a detected feature is a crater is modified based on its associated diameter-range (not compared to the resolution of the image currently used in the multi-resolution setup, but according to classification into one of diameter-ranges - see below; Section 3.2 the distribution of craters from corresponding catalogues). These steps are repeated for all radii and for all data resolutions. Finally, we perform slip-tuning of the crater-candidates, and removal of multiple detections. Because the previous version of CDA (Salamunićcar and Lončarić, 2010a) was used only for Mars, the planetary-radius had been hard-coded. In the new version, it becomes one parameter, so that the same CDA can be used for Mars, the Moon, and potentially for other planetary bodies. This value is used inside the CDA for the computation of slopes and diameter-range of crater according to its size. The proposed CDA can use various edge detection methods such as Canny (1986) and Shen and Castan (1992). The experiments conducted in this work are based on the Canny (1986) edge detection method, and have shown considerably improved crater detection.

The proposed DEM-based CDA is based on our previous CDA (Salamunićcar and Lončarić, 2010a). It has been improved using a specially developed method for interpolation of DEM and parameter-space data, which is suitable for detection of small craters. The most commonly-used interpolating functions are: nearest neighbour, bilinear, bicubic, quadratic splines, cubic B-splines, higher-order B-splines, Catmull-Rom cardinal splines, Gaussians, and truncated sinc (Zitová and Flusser, 2003). However, in the case of their application to CDAs, we should consider that: (1) the optimal implementation of interpolation should be computed in runtime only for required DEM coordinates, as opposed to a static interpolation of the whole data set (this reduces computational complexity and memory requirements); (2) the interpolation itself should not introduce artifacts that will decrease the accuracy or precision of the measurements obtained via CDA (e.g. compiled depth/diameter values); and (3) for the smallest detectable craters we have sparse sampling, wherein each crater is represented with

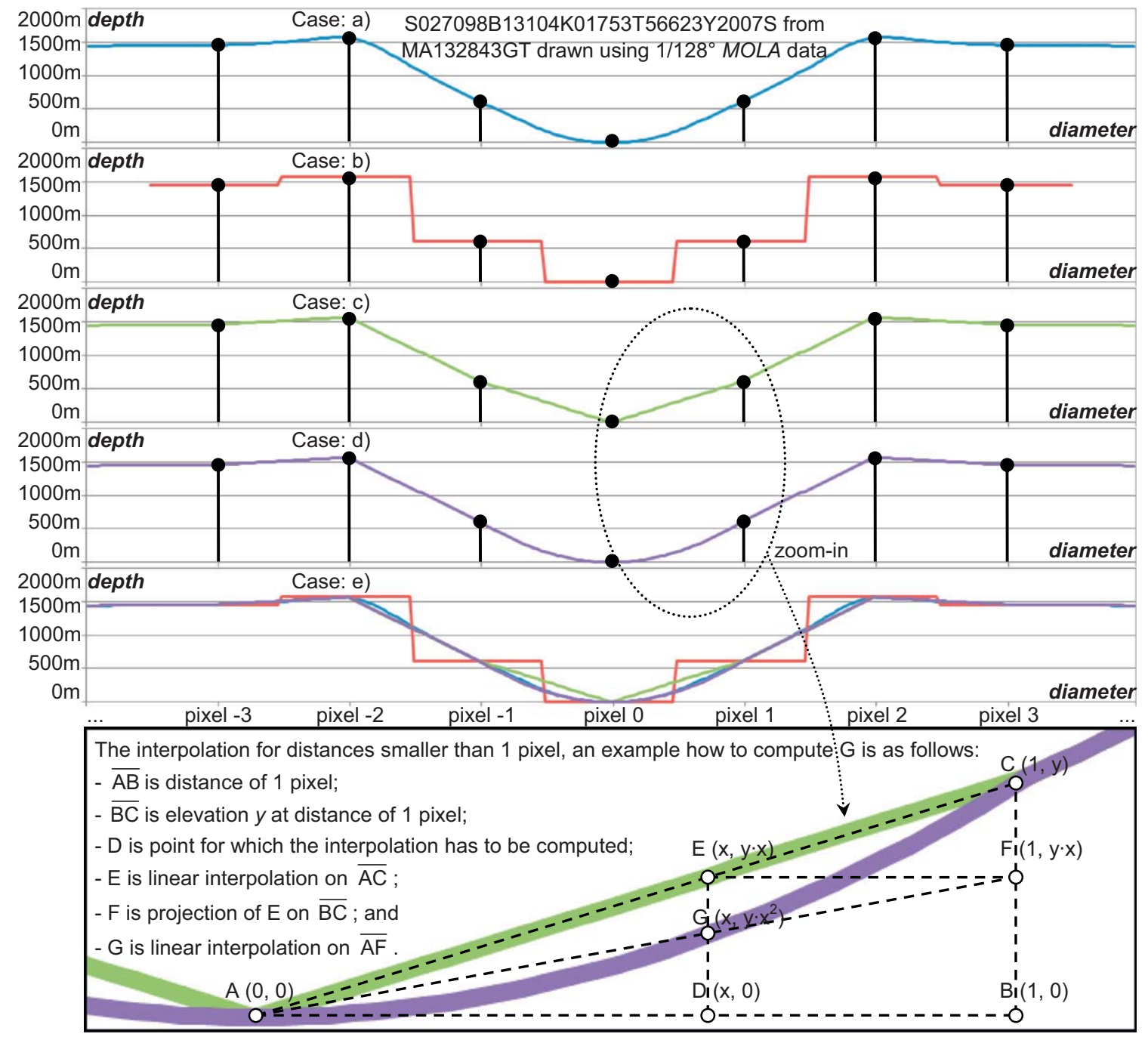

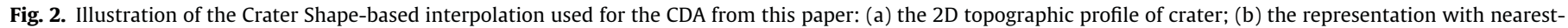

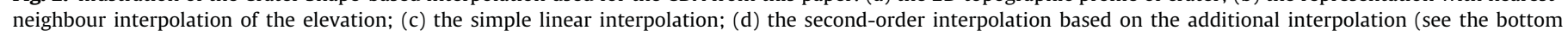

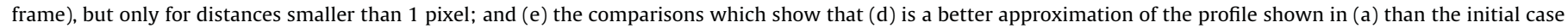
(b) and previous case (c). 
a small number of pixels. On the other hand, the importance of good interpolation lies in: (1) better detection capabilities, particularly for small craters; and (2) more accurate measurements of crater properties. Based on these considerations, we propose the following solution.

Crater Shape-based interpolation used for our CDA is illustrated in Fig. 2. Frame (a) shows the topographic profile of a crater from MA132843GT. Frame (b) shows a pixel representation (known also as the nearest neighbour function), whose pixel size with artificially degraded resolution illustrates the effect of the digitalization of input values. It also enables us to see how various interpolation methods compare to the original full-resolution data. The result of simple linear interpolation is shown in frame (c). It shows an improvement for most segments, except very close to the crater centre. In such cases there is a decrease of accuracy (the curve shown in frame (c) has a first derivative discontinuity at the crater centre, which is not the case for the curve shown in frame (a)). In order to overcome this interpolation artifact, we modify the interpolation between the crater centre and the nearest pixels, as shown in frame (d). The additional interpolation is shown in the bottom frame of Fig. 2. It assumes a parabolic crater shape, which is the case for the majority of small (bowl-shaped) craters, and which is also appropriate for larger craters with central peaks or pits (concave vs. convex parabola). As shown in frame (e), this approximates original (full-resolution) values in all segments considerably better than previous cases (b) and (c). At the same time, approach (d) is computationally very efficient, because simple linear interpolations are computed in only two stages, the second of which is only executed within a one-pixel distance to the crater centre. Even within a one-pixel distance to the crater centre, this is considerably faster than alternative, more complex interpolation approaches (e.g. the computation of coefficients of higher degree polynomials). Therefore, we selected approach (d) for our CDA.

\subsection{Utilisation of method for re-projection of datasets and catalogues}

In previous work (Salamunićcar et al., 2011b), we provided an orthographic projection view with the crater of interest in the centre for better evaluation capabilities of northern-most and southern-most craters. This approach was sufficient for Mars where all the craters from MA130301GT (Salamunićcar et al., $2011 \mathrm{~b}$ ) are located between $87^{\circ} \mathrm{N}$ and $87^{\circ} \mathrm{S}$. However, this is not the case for the Moon, where there are no polar-layer-deposits, and where craters exist at the most extreme polar latitudes. In addition to orthographic projections, we perform a rotation of the entire DEM and optical global datasets, such that the geographical North and South Poles are rotated on the equator of the new coordinate system. The method works as follows: (1) a global dataset is re-projected from the simple cylindrical projection to the sphere; (2) the sphere is rotated by a parameterized amount around the $x, y$, and $z$ axes; (3) the global dataset is re-projected back from the sphere to the simple cylindrical projection. The same method can be used for crater locations, in both directions: (1) to convert geographical crater coordinates to comply with the re-projected datasets; and (2) to convert the rotated coordinates back into the original form, to comply with the initial datasets.

\subsection{Datasets used for crater detection and evaluation}

For Mars, we used datasets prepared in our previous work (Salamunićcar et al., 2011b). For the Moon, we used the $1 / 16^{\circ}$ SELENE LALT (Araki et al., 2009) and up to $1 / 512^{\circ}$ LRO LOLA (Smith

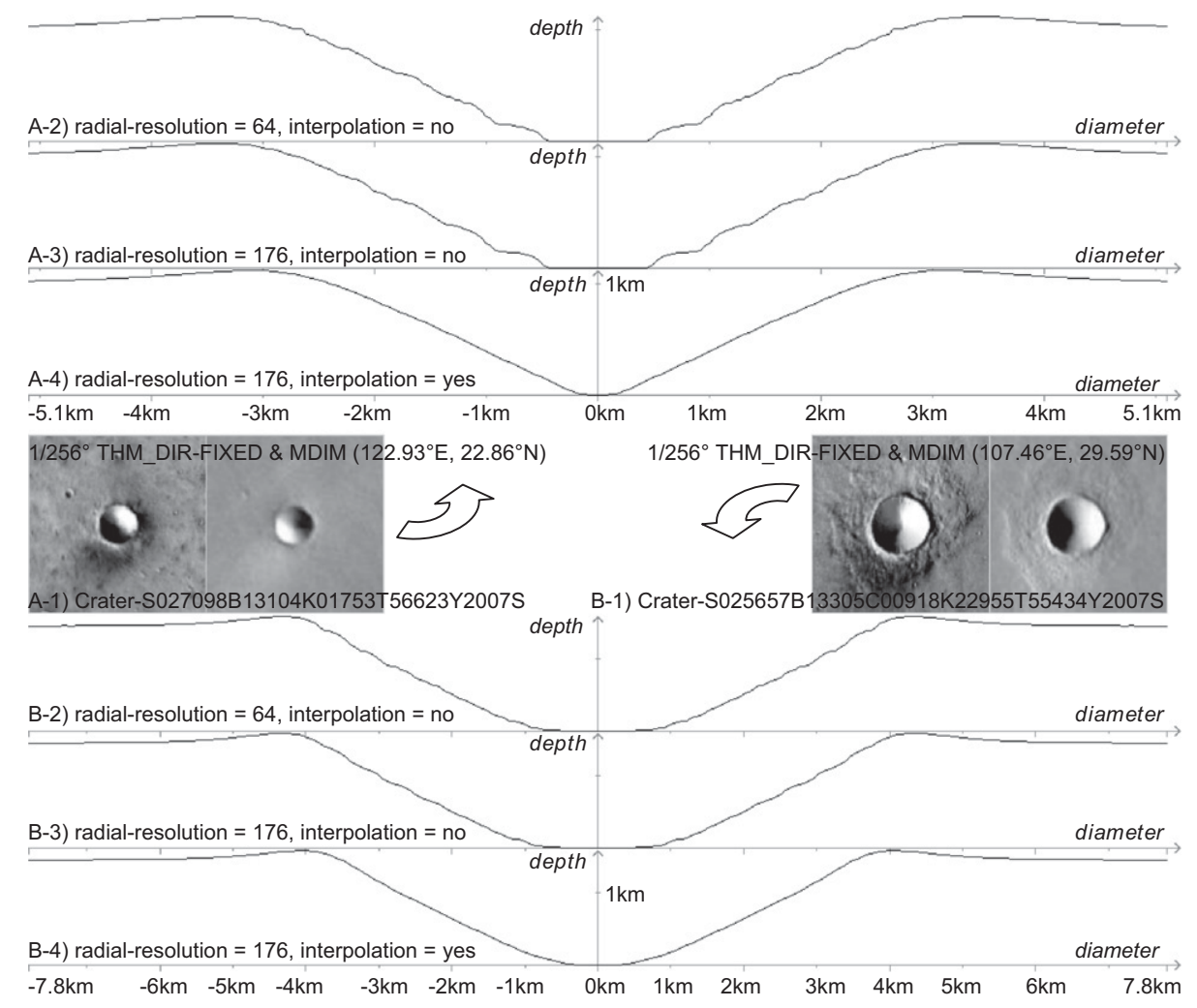

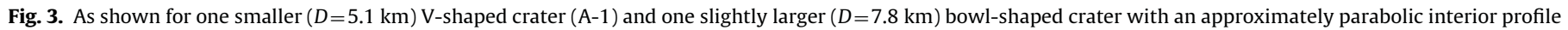

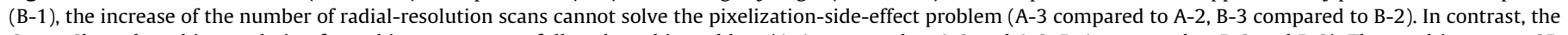

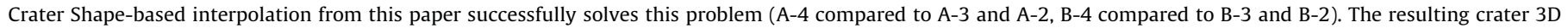
shape and 2D topographic profiles (A-4 and B-4) show no artifact resulting from the sub-optimal data-processing. 
et al., 2010) global DEMs, digitised at 1-m vertical resolution. Unlike previous laser altimeters, which mapped planetary bodies, such as Mars orbiter laser altimeter (MOLA), LOLA is a five-beam laser altimeter with a firing rate of $28 \mathrm{~Hz}$, providing up to 140 measurements per second. The five-beam pattern was designed to characterize the lunar surface at scales relevant to human and robotic exploration, and it translates to an unprecedented alongtrack measurement spacing of $\sim 10 \mathrm{~m}$ (compared to about $1.7 \mathrm{~km}$ for SELENE). LOLA continues to operate, improving its coverage (especially, reducing its average longitudinal gap between tracks). The global Lunar optical image mosaics used during the registration of new craters in the GT catalogue are: $1 / 64^{\circ}$ LASGW (Lunar Airbrushed Shaded Relief Warped to ULCN2005); 1/256 ${ }^{\circ}$ CLEMBASE (Clementine Basemap Mosaic version 2); and 1/512 $L O M$ (Lunar Orbiter Mosaic). In the case of LALT and LOLA DEM datasets and $L A S G W$ optical dataset, post-processing tasks includes only down-sampling and up-sampling to other resolutions, and rendering of optical BMP files. For CLEMBASE, we performed an interpolation fix (as done for Martian THEMIS-DIR) and brightness/contrast correction fixes (as done for Martian MOC). The LOM dataset was optimised for memory usage purposes.

\subsection{Previous catalogues of Lunar craters}

Two Lunar catalogues developed by previous researchers are: (1) McDowell (2007) catalogue, which contains 8639 (named) craters (hereafter LU8639N); and (2) Rodionova et al. (1987) catalogue (hereafter LU14923R), which is the most complete catalogue of Lunar craters prior to this work, containing 14,923 craters (1394 identified by name). An additional Lunar catalogue (Head et al., 2010; Kadish et al., 2011), which contains 5185 craters (hereafter LU5185H) was developed very recently, and was not available when we had completed LU58357GT released earlier in order to facilitate the use of LRO data to survey and estimate impact melt volumes in small- to medium-sized craters (Mazarico et al., 2011). LU5185H was thus included in LU60645GT.

\section{Results and discussion}

In order to evaluate the performance of the proposed CDA, according to the framework for evaluation of CDAs (Salamunićcar et al., 2011b), the resulting new catalogues MA132843GT and LU60645GT are used in addition to the previously assembled catalogue MA57633GT (Salamunićcar and Lončarić, 2008b). The results and discussion are given in the following order:

- improved crater detection algorithm based on new Crater Shape-based interpolation;

- assembled new catalogues MA132843GT and LU60645GT of Martian and Lunar craters; and

- differences between Martian and Lunar craters in depth/ diameter ratio and 2D profiles.

Section 3.1 additionally includes: (1) interpolation-based improvements in 3D-shapes of craters and 2D-topography-profiles; and (2) evaluation of achieved performance of interpolationbased crater detection algorithm. Section 3.2 additionally includes: (1) verification of assigned coordinates and diameters to craters from polar-regions; (2) typical newly catalogued craters from MA132843GT and LU60645GT catalogues; (3) statistics of LU60645GT and MA132843GT catalogues; and (4) advances in integration of diverse morphological descriptors from previous catalogues. Section 3.3 describes one possible application of crater catalogues - insight into differences between Martian and Lunar craters.

\subsection{Improved crater detection algorithm based on new Crater Shape-based interpolation}

The Crater Shape-based interpolation used within the CDA results in improved crater 3D-shapes and 2D topographic profiles, as shown in Fig. 3 for two fresh craters with large depth/diameter ratios. The curve shown in Fig. 2 frame (c) has a discontinuation of first derivation at the crater rim as well (and not only at the crater centre as previously discussed), as well as the curve shown in frame (d), while for the curve shown in frame (a) this is not the case. From this perspective, another important observation is that the discontinuity of the first derivative at the crater rim does not appear in Fig. 3. This is so because this problem has been solved by averaging

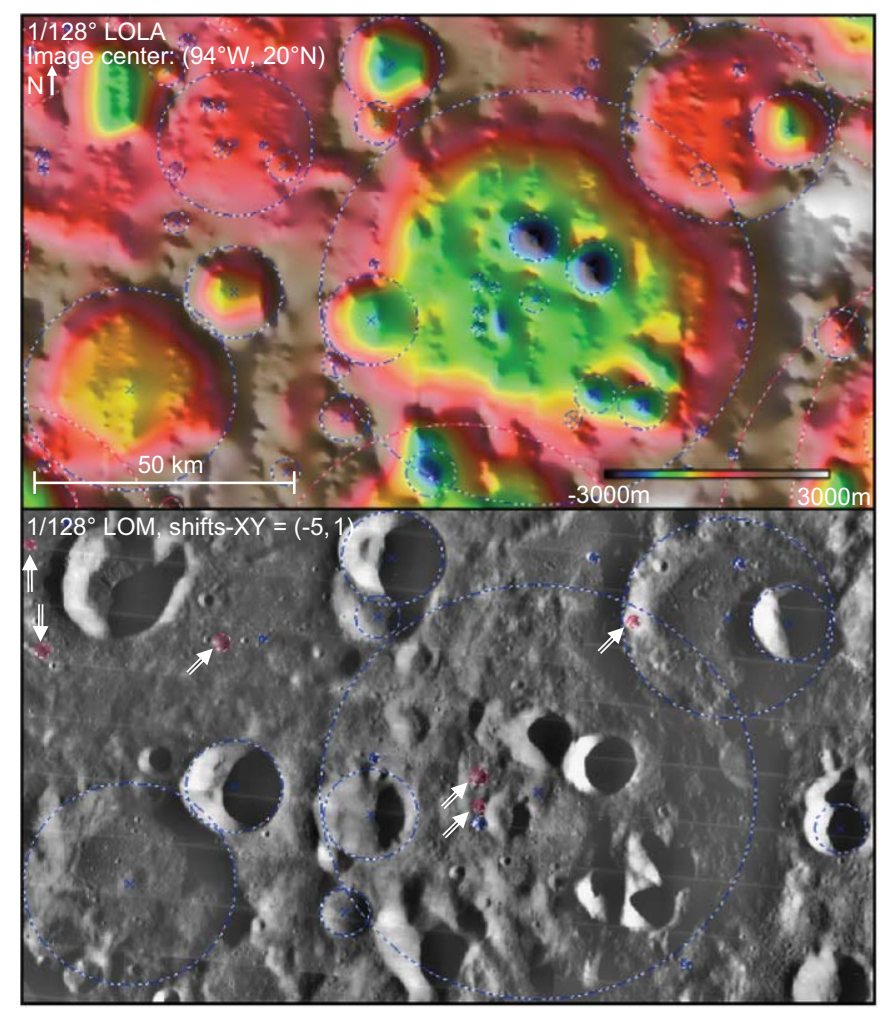

Fig. 4. Part of the $1 / 128^{\circ}$ LOLA dataset and output of our Crater Shape-based interpolation CDA within (blue) and outside (red, not shown in the bottom frame) the selected region (top); crater-candidates with the assigned probability larger than the used threshold which defines those manually evaluated, true (blue) and false (red, labelled with white arrow-pointers) detections, according to the automated verification using LU60645GT (bottom). (For interpretation of the references to color in this figure legend, the reader is referred to the web version of this article).

\section{Table 1}

Definitions and graphs ( $\mathrm{TP}=$ True Positives; $\mathrm{FP}=$ False Positives; $\mathrm{FN}=$ False Negatives; $\mathrm{GT}=$ Ground Truth; $\mathrm{TDR}=$ True Detection Rate; FDR =False Detection Rate; ROC = Receiver Operating Characteristics; F-ROC = Free-response ROC; ROC' $=$ the closest approximation that can be achieved for ROC; $\mathrm{Q}=$ Quality percentage) used for evaluation of CDAs (Salamunićcar et al., 2011b).

\begin{tabular}{ll}
\hline Used definitions & $\begin{array}{l}\text { Used graphs } \\
\text { (horizontal range/vertical range) }\end{array}$ \\
\hline $\mathrm{GT}=\mathrm{TP}+\mathrm{FN}$ & $\mathrm{F}-\mathrm{ROC}[\mathrm{TP} / \mathrm{FP}]$ \\
$\mathrm{TDR}=\mathrm{TP} /(\mathrm{TP}+\mathrm{FN})=\mathrm{TP} / \mathrm{GT}$ & $\mathrm{ROC} \cdot[\mathrm{TDR} / \mathrm{FDR}]$ \\
$\mathrm{FDR}=\mathrm{FP} /(\mathrm{TP}+\mathrm{FP})$ & $\mathrm{Q}[\mathrm{Q} / \mathrm{FDR}]$ \\
$\mathrm{Q}=\mathrm{TP} /(\mathrm{TP}+\mathrm{FP}+\mathrm{FN})=\mathrm{TP} /(\mathrm{GT}+\mathrm{FP})$ & \\
\hline
\end{tabular}


a large number of profiles (we average 176 profiles at different azimuths/angles of the same crater, partially because our CDA searches for craters in radius range between 2 and 28 pixels and in such case the length of rim in pixels is $28 \times 2 \pi=\sim 176$ ).

The difference compared to the CDA from previous work (Salamunićcar and Lončarić, 2010a), which utilised only the Martian DEM $(M O L A)$ is that in this work the Lunar DEMs have been used ( LALT, LOLA) as well. The proposed interpolation-based CDA can successfully detect Lunar craters as shown in Fig. 4: (1) most of the craters are successfully detected; (2) the number of false-detections is low; and (3) coordinates and diameters are precisely defined for a large majority of the craters. As shown, for this region $(x 1=$ “-96.578132" $y 1=$ "20.195315" $x 2=$ " $-90.929678 ” \quad y 2=$ "16.921865"; $x 1$ and $x 2$ are longitudes, $y 1$ and $y 2$ are latitudes of the corners, in the east/planetocentric coordinate system) there are 17 true detections and 6 false detections. All false detections have a diameter smaller than $3.317 \mathrm{~km}$ and for larger craters there are no false detections within this region. For parameterization of the interpolation-based CDA proposed with this paper, we used the planetary radius value for Mars of $3390 \mathrm{~km}$, and for the Moon $1737.4 \mathrm{~km}$.

The evaluation was performed using the framework for evaluation of CDAs (for definition see Table 1) from previous work
(Salamunićcar et al., 2011b), using F-ROC (Salamunićcar and Lončarić, 2008a) and ROC' (Salamunićcar et al., 2011b). The ROC' evaluations for Mars show that the proposed CDA is better than all other available CDAs, according to the area under the ROC' curve criterion (AUROC' is $50.1 \%$ for the CDA by Stepinski and Urbach (2008), 54.0\% for the CDA by Salamunićcar and Lončarić (2010a), and $57.4 \%$ for the proposed interpolation-based CDA). The ROC' evaluations based on lunar datasets (shown in Fig. 5 bottom) show that the proposed interpolation-based CDA is also better for Lunar datasets than the CDA from the previous work (Salamunićcar and Lončarić, 2010a), while other CDAs have not been used before for global processing of Lunar DEM.

In order to compare the relative improvements of overall achieved performance between our previous CDA (Salamunićcar and Lončarić, 2010a) and the CDA from this paper (wherein the only difference is in the usage of Crater Shape-based interpolation) with the improvements between the CDA from Stepinski and Urbach (2008) and our previous CDA (Salamunićcar and Lončarić, 2010a), the following method was used. Let us suppose that CDA-A, CDA-B, and CDA-C achieved performance $A, B$ and $C$, wherein: (1) each value is in the normalised range between 0 and $100 \%$; (2) larger value means better performance; and (3) $A<B$ and $B<C$. In this case, for CDA-B possible improvement is $(1-A)$
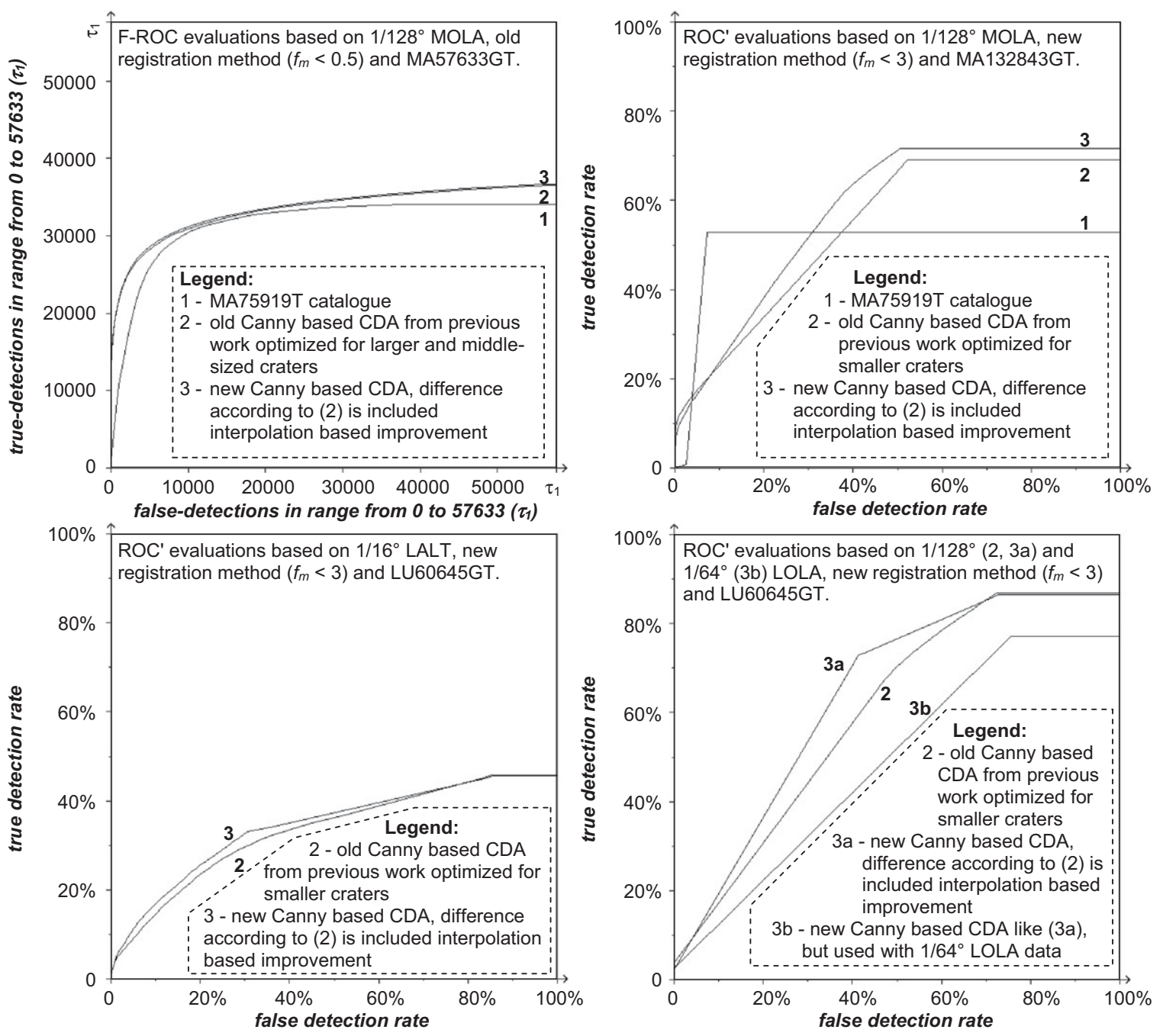

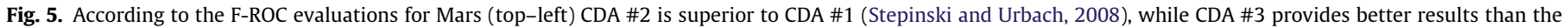

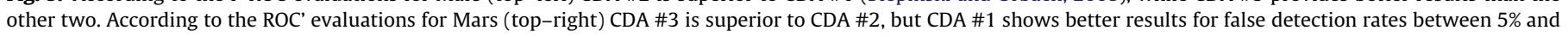

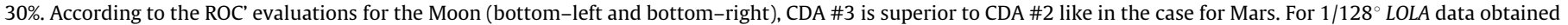
results are better than for $1 / 16^{\circ} L A L T$ or $1 / 64^{\circ} L O L A$ data. 
and achieved improvement is $(B-A)$, while for CDA-C possible improvement is $(1-B)$ while achieved improvement is $(C-B)$. Using this, the ratio between achieved and possible improvement is $R_{B}=(B-A) /(1-A)$ for CDA-B and $R_{C}=(C-B) /(1-B)$ for CDA-C. At the end, these two values are compared, having $I_{B}=R_{B} \cdot 100 /$ $\left(R_{B}+R_{C}\right)$ and $I_{C}=R_{C} \cdot 100 /\left(R_{B}+R_{C}\right)$. For AUROC' values from the previous paragraph, computed values are $50.6 \%$ and $49.4 \%$. Similarly, we can compute for AUFROC ${ }_{1 \tau}$, in which case we are not interested in areas under F-ROC for a horizontal range larger than $1 \tau$ ( $\tau$ is the number of craters in GT catalogue) because in such a case the number of false detections is always larger than the number of correct detections. In order to take into account the fact that some craters from MA132843GT catalogue cannot be detected by either CDA because of dataset limitations, an additional GT catalogue was created from which 19,886 craters not detectable by either CDA have been removed. The overall results are shown in Fig. 6, wherein we also averaged the results from individual computations in order to have as realistic as possible a comparison of achievable performances between different CDAs.

\subsection{Assembled new catalogues MA132843GT and LU60645GT of Martian and Lunar craters}

The results of the method for re-projection of datasets and catalogues are shown in Fig. 7. Using this method, the coordinates and diameters of 22 Lunar craters were corrected. After that,

\begin{tabular}{c|cc|cc|cc|} 
& \multicolumn{2}{|c|}{ AUFROC $_{1 \tau}$} & \multicolumn{2}{c|}{ AUROC' } & \multicolumn{2}{c|}{ Average } \\
\hline MA132843GT & & & & & & \\
& $48.5 \%$ & $51.5 \%$ & $50.6 \%$ & $49.4 \%$ & $49.6 \%$ & $50.4 \%$ \\
\hline $\begin{array}{c}\text { MA132843GT } \\
\text { reduced to } \\
112957 \text { craters }\end{array}$ & $32.9 \%$ & $67.1 \%$ & $51.5 \%$ & $48.5 \%$ & $42.2 \%$ & $57.8 \%$ \\
\hline Average & & & & & & \\
\hline
\end{tabular}

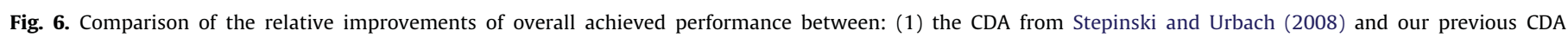

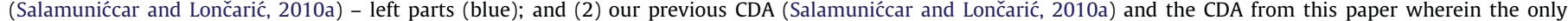

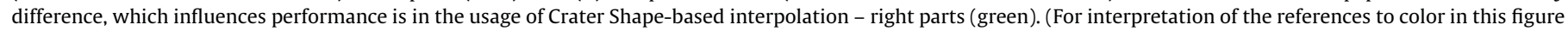
legend, the reader is referred to the web version of this article).

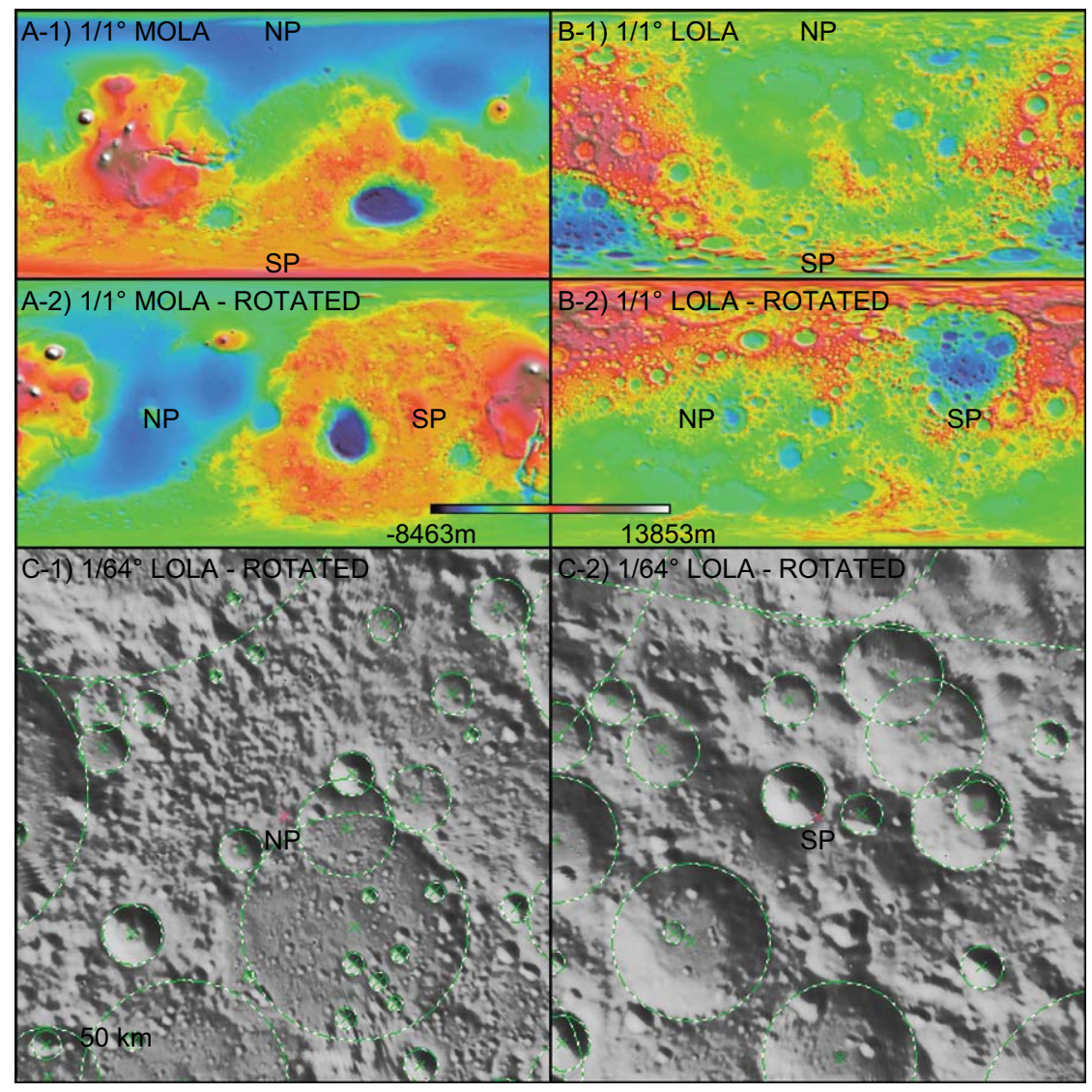

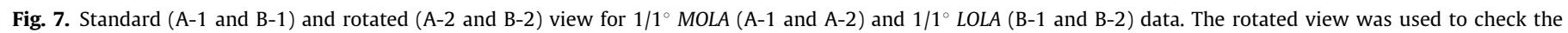
accuracy of assigned coordinates and diameters of craters, as shown for 1/64 LOLA (C-1 and C-2) North-Pole (C-1) and South-Pole (C-2). 
we checked the 50 northern-most and 50 southern-most craters, in order to confirm that additional corrections are not required for LU60645GT. A similar check was performed for MA132843GT, wherein corrections have not been required.

To extend the GT catalogues, we used the framework for evaluation of CDAs from previous work (Salamunićcar et al., 2011b), where all crater-candidates proposed by our CDA are manually evaluated for rejection, or correction of coordinates and diameter and inclusion into the GT catalogues. The main phases of the new work on GT catalogues for Mars and the Moon are shown in Fig. 8. We rejected some of the crater-candidates before manual evaluation, using the framework for evaluation of CDAs from previous work (Salamunićcar et al., 2011b). In the first step (Mars - $1 / 128^{\circ} M O L A$ ), we found the maximum of $\mathrm{Q}$ (Quality percentage) curve (for definition see Table 1) using MA130301GT

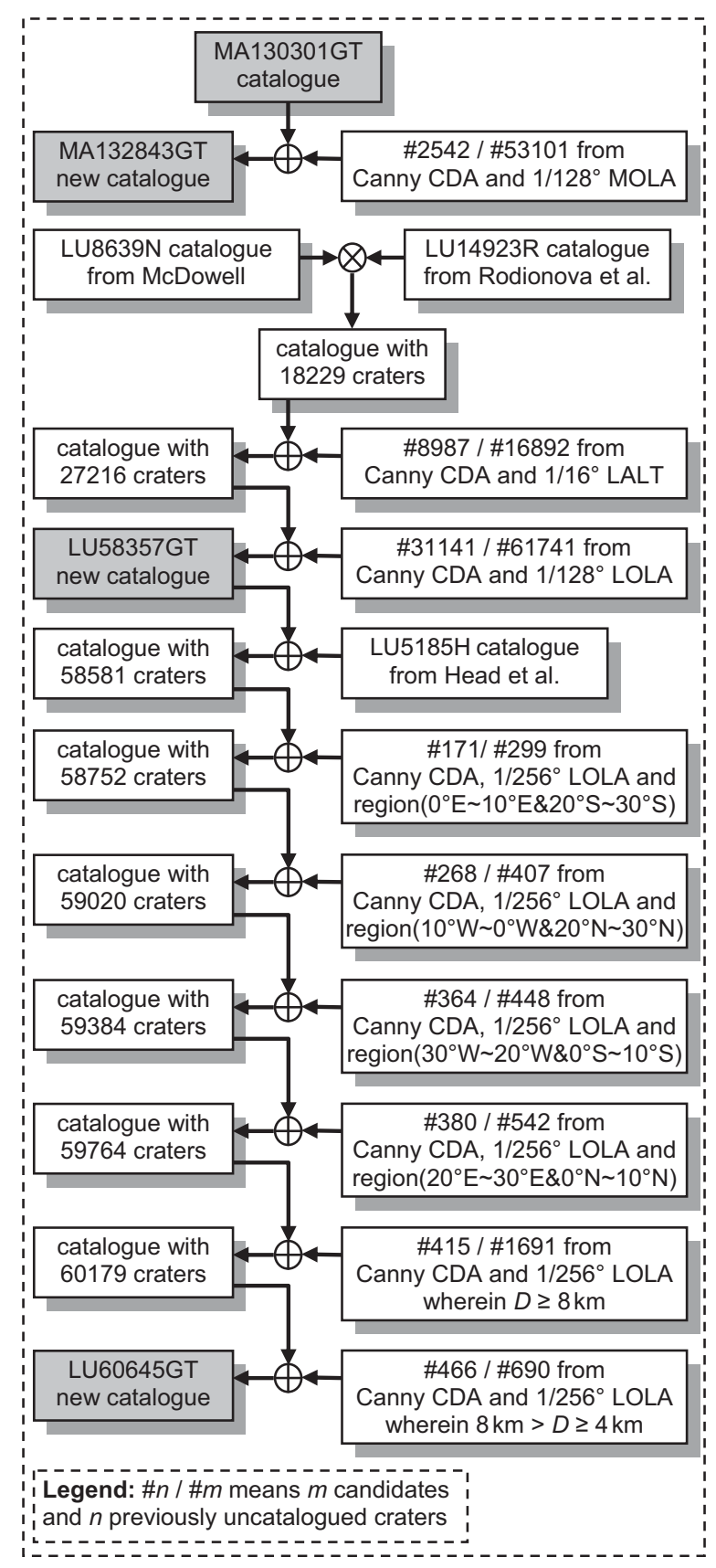

Fig. 8. Main phases of the new work on GT catalogues for Mars (top) and the Moon (bottom) in which the new work is based on the interpolation-based CDA. and the associated probability threshold $\sim 0.3907$ (hereafter $\mathrm{t}$ ) and removed all crater-candidates with associated probabilities smaller than t. The recomputed $t$ for MA132843GT is the same number. The value of $t$ computed for Mars has been used for Lunar catalogues as well because it was obtained using GT catalogue, which was much more complete than that available for the Moon at that time. When we assembled LU60645GT we recomputed the Lunar value of $t$. It is remarkably similar to the previously computed for Mars (difference is only of the order $10^{-5}$ ). This is explainable by completeness of LU60645GT in comparison with MA132843GT and the robustness of the interpolation-based CDA proposed by this paper, regarding detection of craters on two different planetary bodies (Moon and Mars).

As shown in Fig. 9 (top), typical new entries in Martian GT catalogue are mostly small craters, and some larger much degraded craters. These are the craters that the new interpolation-based CDA from this paper successfully detected, which was not the case for our previous CDA (Salamunićcar and Lončarić, 2010a). As shown in Fig. 9 (bottom), there are many more new entries in Lunar GT catalogue than in the Martian one. The reason is that preceding Lunar catalogues were considerably less complete than the preceding MA130301GT Martian catalogue.

The distribution of craters from corresponding catalogues and classification into one of diameter-ranges is given in Fig. 10. The completeness is estimated using the Craterstats (Michael, 2010) programme. The flattening of lines at small diameters is related to the input dataset resolution. The CDA detected numerous small craters $(D<8 \mathrm{~km})$ in the LOLA dataset, which could not be verified due to inadequate high-resolution imagery coverage, and therefore has not been included into LU60645GT. In addition, we expect a correlation between poorer imagery coverage and reduced density of small craters $(D<8 \mathrm{~km})$ (e.g. fewer smaller detected craters validated on the farside or higher-latitudes, where imagery coverage was worse).

The catalogue integration is useful for researchers interested in craters on Mars and the Moon. The new catalogue LU60645GT is a superset of the previous Lunar catalogues, as shown in Fig. 11. With our approach, LU60645GT contains everything that was included in these catalogues, plus: (1) the correlation between various morphological descriptors from the catalogues used; (2) the correlation between manually assigned attributes and automated depth/diameter measurements from LU60645GT by our CDA; (3) average errors and their standard deviations for manually and automatically assigned attributes, such as position coordinates, diameter, depth/diameter ratio, etc; and (4) positional accuracy of features in the used datasets, wherein our catalogue contains 60,645 cross-references between each of the used datasets. Additionally, surface dating could potentially be improved with the exhaustiveness of this new catalogue. The same kind of contribution was also made in the case of the new Martian catalogue MA132843GT, as described into more detail in the previous work for MA130301GT (Salamunićcar et al., 2011b).

\subsection{Differences between Martian and Lunar craters in depth/ diameter ratio and $2 \mathrm{D}$ profiles}

The Crater Shape-based interpolation CDA from this paper can be used in combination with the new LU60645GT and MA132843GT catalogues of Lunar and Martian impact craters in order to provide automated morphometry measurements of depth/diameter ratio, topographic-cross-profiles, etc. (Salamunićcar and Lončarić, 2009, 2010a). Now that catalogue LU60645GT exists, in addition to MA132843GT (extension of the previously available MA130301GT), it is possible to compare morphometry measurements of Martian and Lunar impact craters. As shown in Fig. 12 (top), the transition of the depth/diameter ratio of the youngest craters on Mars occurs 


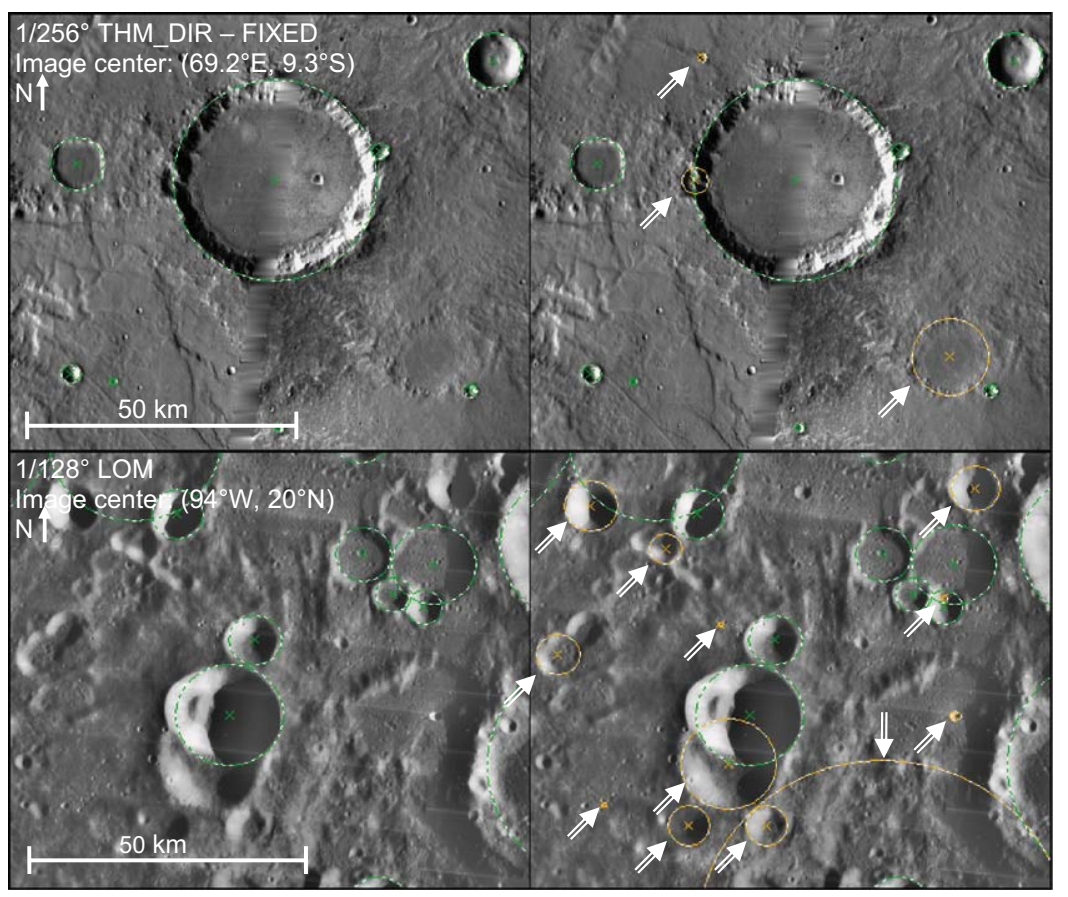

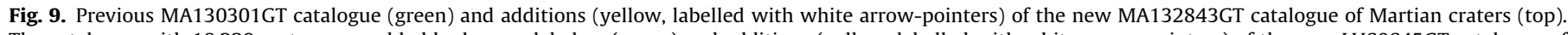

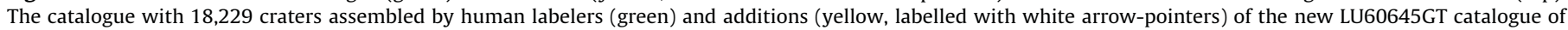
Lunar craters (bottom). (For interpretation of the references to color in this figure legend, the reader is referred to the web version of this article).
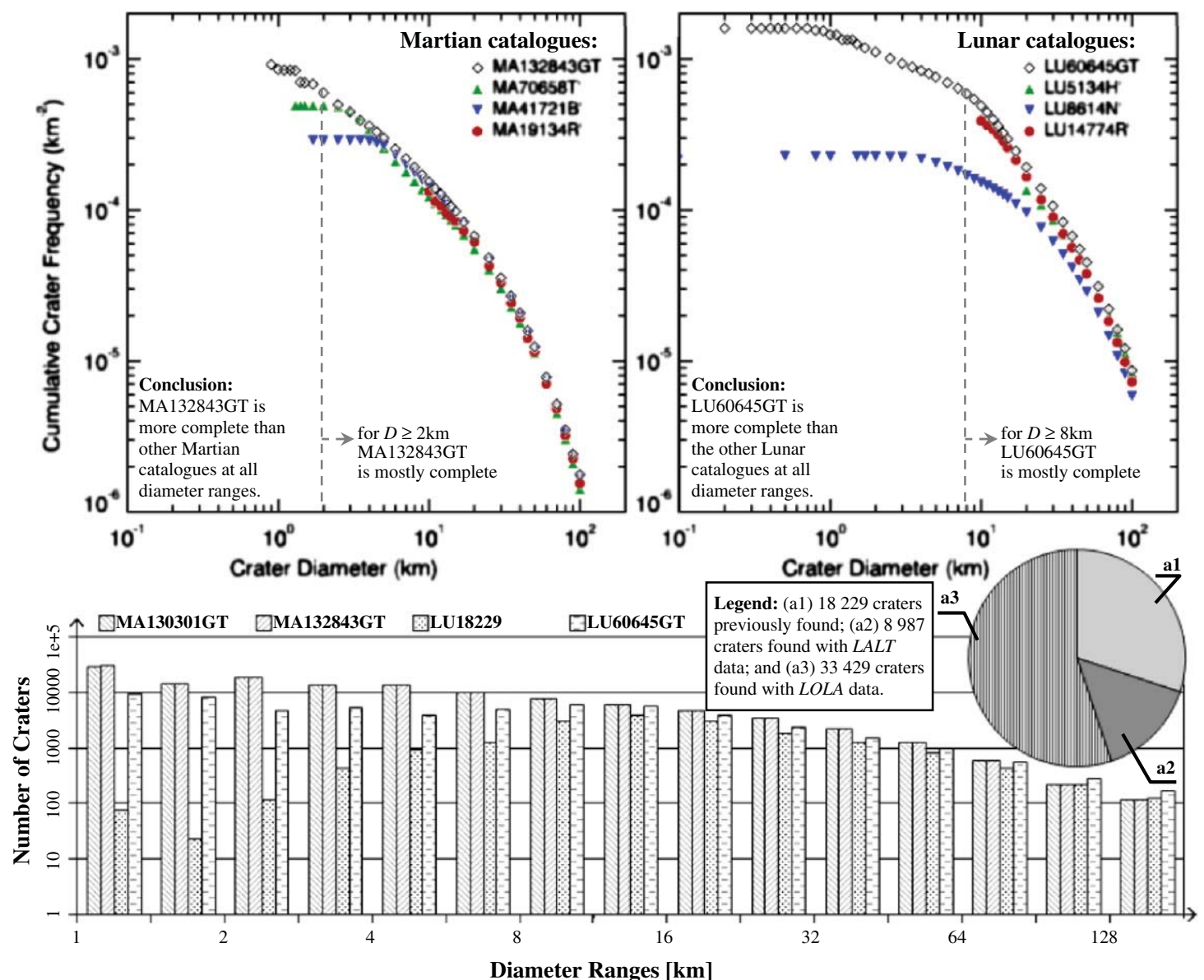

\begin{tabular}{l|c|c|c|c|c|c|c|c|c|c|c|c|c|c|c|} 
MA130301GT & 29700 & 14818 & 19888 & 14026 & 13848 & 10382 & 8225 & 6448 & 4895 & 3512 & 2308 & 1304 & 603 & 227 & 117 \\
MA132843GT & 31630 & 15129 & 20073 & 14059 & 13876 & 10394 & 8238 & 6464 & 4901 & 3518 & 2309 & 1305 & 603 & 227 & 117 \\
LU18229 & 78 & 23 & 122 & 456 & 972 & 1316 & 3165 & 4026 & 3194 & 1936 & 1311 & 816 & 459 & 229 & 126 \\
LU60645GT & 9755 & 8758 & 5005 & 5439 & 3994 & 5332 & 6282 & 5908 & 4069 & 2471 & 1606 & 1006 & 568 & 283 & 169
\end{tabular}

Fig. 10. An assessment of completeness of the catalogues and the distribution of craters from LU60645GT regarding the contributions. 


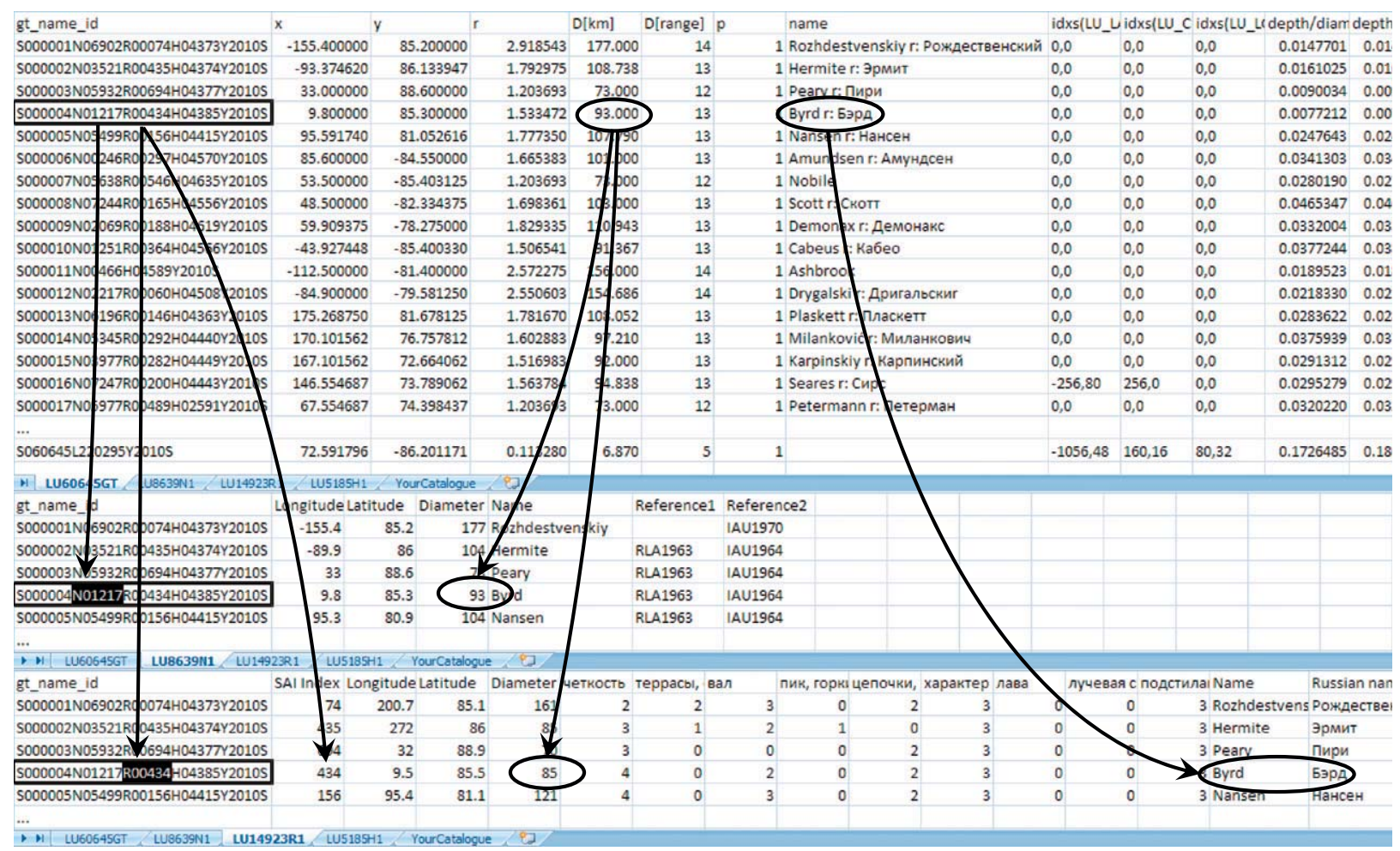

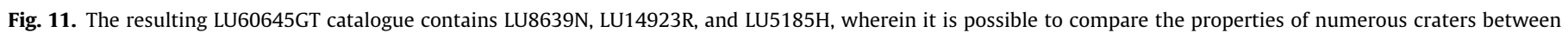
catalogues.

at $D=5.8 \mathrm{~km}$, while on the Moon it occurs at $D=18.4 \mathrm{~km}$. According to its diameter, we can classify each crater into one of the seven following ranges: (1) $1 \mathrm{~km} \leq D<2 \mathrm{~km}$; (2) $2 \mathrm{~km} \leq D<4 \mathrm{~km}$; (3) $4 \mathrm{~km} \leq D<8 \mathrm{~km}$; (4) $8 \mathrm{~km} \leq D<16 \mathrm{~km}$; (5) $16 \mathrm{~km} \leq D<32 \mathrm{~km}$; (6) $32 \mathrm{~km} \leq D<64 \mathrm{~km}$; and (7) $64 \mathrm{~km} \leq D$. As shown in Fig. 12 (centre), it is possible to compare average topography-cross-profiles of Lunar and Martian craters. They follow the trend of the youngest craters, wherein for Lunar craters depth/diameter ratio and height of crater rim is approximately two times larger than for Martian craters. For each average topographic-cross-profile, using $d / D$ ratio, all craters from a selected range are in addition classified according to whether they have higher or lower $d / D$ ratio. The whole procedure is recursively repeated 3 times. The result is that the craters within each diameter range are additionally classified into 8 sub-groups according to their $d / D$ value. As shown in Fig. 12 (bottom), the above trend is consistent for all ranges from the freshest to the most heavily degraded/eroded craters.

\section{Conclusions}

The main results of this paper are: (1) the new catalogue LU60645GT of Lunar craters (complete up to $\sim D \geq 8 \mathrm{~km}$ ), which contains a considerably larger number of craters and associated attributes than any other currently available catalogue of Lunar craters; (2) the new catalogue MA132843GT of Martian craters (an expansion of the previous MA130301GT), which is the most complete (up to $\sim D \geq 2 \mathrm{~km}$ ) publicly available catalogue of Martian craters; (3) considerable improvement of previous CDA (Salamunićcar and Lončarić, 2010a) using new Crater Shapebased interpolation method from this paper, wherein the improved CDA was successfully used during work on LU60645GT and MA132843GT. As previously achieved for Mars with our previous catalogue MA130301GT (Salamunićcar et al., 2011b) and catalogue MA132843GT from this paper, for the Moon the new LU60645GT catalogue provides everything that was provided by the previous Lunar catalogues, plus numerous new possibilities as enumerated in Section 3.2. The accompanying results of this paper are: (1) the possibility of comparing Lunar and Martian craters regarding depth/diameter ratio, 2D profiles, etc., wherein a considerably larger number of craters than available from the previous work leads to globally more representative, statistically more significant, more precise results; (2) utilisation of a method for the re-projection of datasets and catalogues, which is very useful for the evaluation and registration of craters that are very close to poles; and (4) the extension of the previous framework for evaluation of CDAs (Salamunićcar et al., 2011b) with datasets and GT catalogue for the Moon, which can be used in parallel with those previously prepared for Mars.

The new Crater Shape-based interpolation module improves our previous CDA as follows: (1) it decreases the number of falsedetections for the required number of true detections, which leads to a smaller number of crater-candidates that need to be evaluated manually and at the same time to larger extensions of GT catalogues with previously uncatalogued craters; (2) it improves detection capabilities for very small craters, which leads to detection of considerably more craters from the same dataset; and (3) it provides more accurate automated measurements of 2D profiles and other crater properties such as depth-diameter ratio and more precise comparison of Lunar and Martian impact craters. In fact, the measure of relative difference in overall achieved performance between the CDAs from our previous work and this paper is $54.1 \%$ (wherein the only difference is in the new Crater Shape-based interpolation module - different from the linear one exclusively for distances smaller than one pixel), which is more than the difference in performance of $45.9 \%$ between the CDA from Stepinski and Urbach (2008) and our previous CDA (where the CDAs are completely different). A possible explanation - based on the presumption that CDA usually looks (in the first approximation) for circles/ellipses wherein all planetary bodies 


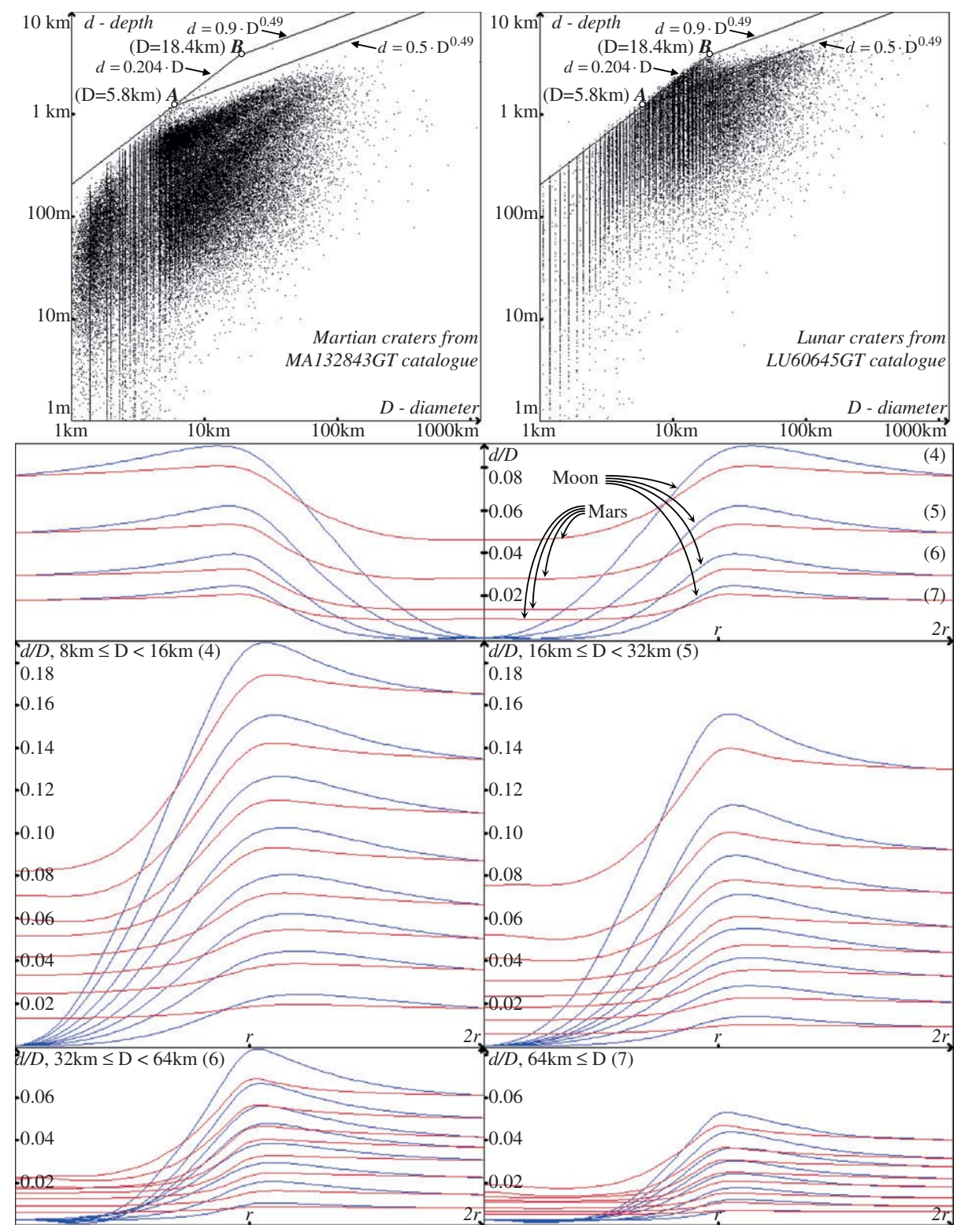

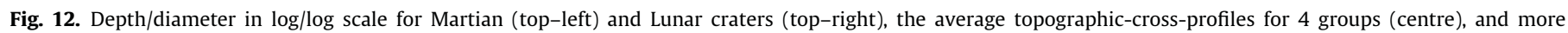
detailed insight (bottom).

have craters with approximately those shapes - is that there is no reason why the same CDA should not work on different bodies. The experience of the other researchers is that this is not the case when the CDA is based on supervised learning, where the issue could also be the application of learning results from one image to another. This led to the investigation of advanced machinelearning techniques such as transfer-learning (Ding et al., 2011). The conclusion from this paper is that differences in shapes between Lunar and Martian craters (e.g. $\sim 2$ times larger/smaller average depth/diameter ratio) could lead to similar problems for other CDAs as well, because of e.g. the over-optimisation problem. From this perspective, instead of only claiming that the same CDA can be used for detection of craters from more than one planetary body, it is advisable to actually demonstrate this, as done in this paper for global DEMs of Mars and the Moon.

The new Lunar crater catalogue from this paper is already in use in the current project of impact melt volume estimates of small- to medium-sized Lunar craters using LRO data (Mazarico et al., 2011). The Crater Shape-based interpolation CDA from this paper is already in use in current projects of cataloguing: (1) Phobos' craters (Salamunićcar et al., 2011c, 2011d); and (2) additional Lunar craters using DEMs reconstructed from optical images (Lončarić et al., 2011, Salamunićcar et al., 2011e). The goals and challenges for future work include integration of MA132843GT with the manually assembled catalogue, which currently contains over 288,993 craters expected to be completed soon (Robbins and Hynek, 2010), and the use of improved LOLA DEMs ( $1 / 256^{\circ}$ and $1 / 512^{\circ}$ currently, up to $1 / 1024^{\circ}$ eventually) in combination with the newly released $L R O$ global image mosaic, in order to improve the Lunar catalogue.

\section{Acknowledgements}

The authors would also like to thank, for permission to use their data, the compilers of the catalogues that we used particularly Jonathan McDowell and Janna Rodionova. 


\section{References}

Araki, H., Tazawa, S., Noda, H., Ishihara, Y., Goossens, S., Sasaki, S., Kawano, N., Kamiya, I., Otake, H., Oberst, J., Shum, C., 2009. Lunar global shape and polar topography derived from Kaguya-LALT laser altimetry. Science 323 (5916), 897-900.

Bandeira, L., Saraiva, J., Pina, P., 2007. Impact crater recognition on Mars based on a probability volume created by template matching. IEEE Transaction on Geoscience and Remote Sensing (12), 4008-4015.

Barlow, N.G., Barnes, C.W., Barnouin-Jha, O.S., Boyce, J.M., Chapman, C.R., Costard, F.M., Craddock, R.A., Garvin, J.B., Greeley, R., Hare, T.M., Kuzmin, R.O. Mouginis-Mark, P.J., Newsom, H.E., Sakimoto, S.E.H., Stewart, S.T., Soderblom, L.A., 2003. Utilizing GIS in Martian impact crater studies. In: Proceedings of the ISPRS WG IV/9 Extraterrestrial Mapping Workshop: Advances in Planetary Mapping. Abstract.

Canny, J.F., 1986. A computational approach to edge detection. IEEE Transaction on Pattern Analysis and Machine Intelligence 8 (6), 679-698.

Cooper, G.R.J., 2003. Feature detection using sun shading. Computers \& Geosciences 29 (8), 941-948.

Cooper, G.R.J., Cowan, D.R., 2004. The detection of circular features in irregularly spaced data. Computers \& Geosciences 30 (1), 101-105.

Ding, W., Stepinski, T.F., Bandeira, L., Vilalta, R., Wu, Y., Lu, Z., Cao, T., 2010. Automatic detection of craters in planetary images: an embedded framework using feature selection and boosting. In: Proceedings of the 19th ACM International Conference on Information and Knowledge Management.

Ding, W., Stepinski, T.F., Mu, Y., Bandeira, L., Ricardo, R., Wu, Y., Lu, Z., Cao, T., Wu, X 2011. Sub-Kilometer Crater Discovery with Boosting and Transfer Learning. ACM Transaction on Intelligent Systems and Technology 2 (4), 1-22.

Flores-Méndez, A., Suarez-Cervantes, A., 2009. Circular degree Hough transform. Lecture Notes in Computer Science 5856, 287-294.

Head, J.W., Fassett, C.I., Kadish, S.J., Smith, D.E., Zuber, M.T., Neumann, G.A., Mazarico, E., 2010. Global distribution of large Lunar craters: implications for resurfacing and impactor populations. Science 329 (5998), 1504-1507.

Kadish, S.J., Fassett, C.I., Head, J.W., Smith, D.E., Zuber, M.T., Neumann, G.A., Mazarico, E., 2011. A global catalog of large Lunar craters $(\geq 20 \mathrm{~km})$ from the Lunar Orbiter Laser Altimeter. In: Proceedings of the 42nd Lunar and Planetary Science Conference, p. 1006. Abstract [CDROM].

Krøgli, S.O., Dypvik, H., 2010. Automatic detection of circular outlines in regiona gravity and aeromagnetic data in the search for impact structure candidates. Computers \& Geosciences 36 (4), 477-488.

Lončarić, S., Salamunićcar, G., Grumpe, A., Wöhler, C., 2011. Automatic Detection of Lunar Craters Based on Topography Reconstruction from Chandrayaan-1 M3 Imagery. In: Proceedings of the 42nd Lunar and Planetary Science Conference p. 1454. Abstract [CDROM].

Mazarico, E., Barnouin, O.S., Salamunićcar, G., Zuber, M.T., 2011. Impact melt volume estimates of small- to medium-sized Lunar craters from Lunar Reconnaissance Orbiter data. In: Proceedings of the 42nd Lunar and Planetary Science Conference, p. 2075. Abstract [CDROM].

McDowell, J., 2004 2007. A merge of a digital version of the list of lunar craters from Andersson and Whitaker with the list from the USGS site. $<$ http://www planet4589.org/astro/lunar/Craters $\rangle$. [accessed 12 April, 2010].

Melosh, H.J., Ivanov, B.A., 1999. Impact crater collapse. Annual Review of Earth and Planetary Sciences 27, 385-415.

Michael, G.G., 2003. Coordinate registration by automated crater recognition. Planetary and Space Science 51 (9-10), 563-568.

Michael, G.G., 2010. Craterstats application. 〈http://hrscview.fu-berlin.de/crater stats.html $>$. [accessed 09 June, 2010].

Robbins, S.J., Hynek, B.M., 2010. Progress towards a new global catalog of Martian craters and layered ejecta properties, complete to $1.5 \mathrm{~km}$. In: Proceedings of the 41st Lunar and Planetary Science Conference, p. 2257. Abstract [CDROM].
Rodionova, J.F., Karlov, A.A., Skobeleva, T.P., Konotopskaya, E.V., Shevchenko, V.V. Kozubskiy, K.E., Dekhtyareva, K.I., Smolyakova, T.F., Tishik, L.I., Fedorova, E.A., 1987. Morphological catalogue of the craters of the Moon. V.V. Shevchenko (Ed.), 〈http://selena.sai.msu.ru/Home/Moon_Cat/Moon_cate.htm $\rangle$. [accessed 26 April, 2005].

Shen, J., Castan, S., 1992. An optimal linear operator for step edge detection. Computer Vision, Graphics, and Image Processing: Graphical Models and Image Processing 54 (2), 112-133.

Salamunićcar, G., Lončarić, S., 2008a. Open framework for objective evaluation of crater detection algorithms with first test-field subsystem based on MOLA data. Advances in Space Research 42 (1), 6-19.

Salamunićcar, G., Lončarić, S., 2008b. GT-57633 catalogue of Martian impact craters developed for evaluation of crater detection algorithms. Planetary and Space Science 56 (15), 1992-2008.

Salamunićcar, G., Lončarić, S., 2009. Automated depth/diameter and topographiccross-profile measurements based on GT-57633 Catalogue of Martian Impact Craters and MOLA Data. In: Proceedings of the 40th Lunar and Planetary Science Conference, p. 1085. Abstract [CDROM].

Salamunićcar, G., Lončarić, S., 2010a. Method for crater detection from Martian digital topography data using gradient value/orientation, morphometry, votesanalysis, slip-tuning and calibration. IEEE Transaction on Geoscience and Remote Sensing 48 (5), 2317-2329.

Salamunićcar, G., Lončarić, S., 2010b. Method for crater detection from digita topography data: interpolation based improvement and application to Lunar SELENE LALT data. In: Proceedings of the 38th COSPAR Scientific Assembly, p. B02-0042-10. Abstract [CDROM]

Salamunićcar, G., Lončarić, S., Mazarico, E., 2011a. From interpolation based crater detection algorithm and LOLA data towards the most complete global catalog of Lunar craters. In: Proceedings of the 42nd Lunar and Planetary Science Conference, p. 1449. Abstract [CDROM].

Salamunićcar, G., Lončarić, S., Pina, P., Bandeira, L., Saraiva, J., 2011b. MA130301GT catalogue of Martian impact craters and advanced evaluation of crater detection algorithms using diverse topography and image datasets. Planetary and Space Science 59 (1), 111-131.

Salamunićcar, G., Lončarić, S., Pina, P., Bandeira, L., Saraiva, J., 2011c. Machine Detection and Global Catalog of Phobos Craters. In: Proceedings ot the 42nd Lunar and Planetary Science Conference, p. 1451. Abstract [CDROM].

Salamunićcar, G., Lončarić, S., Pina, P., Bandeira, L., Saraiva, J., 2011d. Integrated Crater Detection Algorithm and Systematic Cataloging of Phobos Craters. In: Proceedings of the 7th International Symposium on Image and Signal Processing and Analysis p. 175, 591-596.

Salamunićcar, G., Lončarić, S., Grumpe, A., Wöhler, C., 2011e. Hybrid Method for Detection of Lunar Craters Based on Topography Reconstruction from Optical Images. In: Proceedings of the 7th International Symposium on Image and Signal Processing and Analysis p. 263, 597-602.

Smith, D.E., Zuber, M.T., Neumann, G.A., Lemoine, F.G., Mazarico, E., Torrence, M.H., McGarry, J.F., Rowlands, D.D., Head III, J.W., Duxbury, T.H., Aharonson, O. Lucey, P.G., Robinson, M.S., Barnouin, O.S., Cavanaugh, J.F., Sun, X., Liiva, P., Mao, D.d., Smith, J.C., Bartels, A.E., 2010. Initial observations from the Lunar Orbiter Laser Altimeter (LOLA). Geophysical Research Letters 37 (L18204), 1-6.

Stepinski, T.F., Urbach, E.R., 2008. Completion of the first automatic survey of craters on Mars. In: Proceedings of the 11th Mars Crater Consortium meeting, US Geological Survey in Flagstaff-AZ.

Vetro, R., Simovici, D.A., 2010. Entropic quadtrees and mining Mars craters. Lecture Notes in Computer Science 6171, 210-221.

Zitová, B., Flusser, J., 2003. Image registration methods: a survey. Image and Vision Computing 21 (11), 977-1000. 